\title{
AN EVENT-BASED APPROACH FOR EXTREME JOINT PROBABILITIES OF WAVES AND SEA LEVELS
}

\author{
Franck Mazas ${ }^{1}$ and Luc Hamm ${ }^{1}$
}

\begin{abstract}
A methodology for determining extreme joint probabilities of two metocean variables, in particular wave height and sea level, is presented in the paper. This methodology focuses in particular on the sampling of the time series, which should be based on the notion of event: either the event generating the variables whose joint probabilities are wanted (such as a storm generating waves and surges) or the event that is a result of the combination of these variables (such as a beach erosion event generated by waves at high sea level). A classification is proposed for multivariate analyses in order to help the choice of the sampling method. The dependence between the variables is analysed using tools such as the chi-plot, of which an enhanced presentation is proposed, then is modelled by extreme-value copulas (Gumbel-Hougaard, Galambos and Hüsler-Reiss) estimated by Canonical Maximum Likelihood or by the upper tail dependence coefficient. Joint return periods are then computed. A comparison is made with a simulation from the JOIN-SEA software on a dataset of wave height and sea levels offshore Brest, France. Then the bivariate methodology is extended to a multivariate framework. The distribution of sea level is determined by an indirect approach (extrapolation of extreme surges then convolution with the astronomical tide) and the dependence is analyzed between the wave height and the surge component only. A bidimensional convolution between the joint distribution of wave height and surge and the distribution of the astronomical tide yields the joint distribution of wave height and sea level. The application of this method to the dataset of Brest and its comparison with the bivariate approach are finally discussed.
\end{abstract}

Keywords: Waves - sea levels joint probabilities; copulas; event

\section{INTRODUCTION}

Coastal flooding events are caused by a combination of high sea levels and large wave heights, though neither of these constituents needs to be extreme. In U.K. and neighboring countries, advanced engineering practice relies actually on an approach involving the four following steps:

1. Data selection / Sampling of the joint time series for extreme analysis

2. Modelling of marginal distributions of Hs and Still Water Level (SWL)

3. Analysis and modelling of the dependence structure (Hs, SWL)

4. Computation of joint probabilities and curves of joint return periods

The software JOIN-SEA (Hawkes et al 2002) is a well-known reference of such an approach. The present study addresses alternatives to original choices implemented in JOIN-SEA.

In step 1, less conventional methods like a) a bivariate threshold and b) POT declustering on a univariate response function depending on sea level, Hs and covariates (e.g. overtopping over a coastal structure) have been explored in addition to the classical high tide sampling. The interest in POT sampling (b) is in the use of covariates and in the link with physical events, as described by Bernardara et al. (2014). In step 2, the marginal distributions are fitted with a mixture model (empirical / parametric) by connecting an empirical distribution for the bulk values to a parametric tail for extreme values (e.g. GPD). In step 3, the use of a copula for extreme values, such as Gumbel copula, has been tested as an alternative to the normal bivariate distribution for modelling dependency between Hs and SWL.

This methodology has been refined by extending the indirect approach for extreme sea levels published by Mazas et al. (2014) to a multivariate analysis modeling the dependence between wave heights and the stochastic surge component only. Then a classical convolution between surge and tide yields the marginal distribution of sea level (possibly accounting for tide-surge dependence), while a 2D-convolution of the joint distribution of $\mathrm{Hs}$ and surge on the one hand, and the distribution of astronomical tide on the other hand, yields a joint distribution of Hs and SWL.

First, the simple version of our methodology (high tide sampling and no separation of sea level) is applied to the case study of Brest, France for which sea level measurements and wave parameters from numerical modeling are available over the period 1953-2010, and compared with JOIN-SEA results, kindly provided by Dr Peter Hawkes (HR Wallingford).

The more sophisticated application of the methodology (sampling by a univariate response function and separate analysis of tide and surge) is then presented, and the difference in the results is discussed.

\footnotetext{
${ }^{1}$ ARTELIA, 6 rue de Lorraine, 38130 Echirolles, France
} 


\section{BIVARIATE METHODOLOGY FOR JOINT OCCURRENCE OF WAVES AND SEA LEVELS}

\section{Sampling}

The sampling of the time series is a critical point of any extreme analysis, be it univariate or multivariate. The analyst usually deals with time series of the variables, observed (i.e. measured or modelled) at regular time steps: they can be called sequential values. Because the physical phenomena have a non-nil duration and a momentum, the sequential values are usually auto-correlated (Walton 2000). The finer the time step and the longer the duration of the physical phenomenon, the more autocorrelated the sequential data. Still, the extreme value theory requires working with an i.i.d. sample. Homogeneous subsets of the time series must thus be identified (in order to consider i.d. data) then declustering selects independent data.

Bernardara et al. (2014) detail a two-step framework in the univariate case. In a first step, the aim is to identify physical events such as a storm within the time series of the sequential observations. Using exceedances over a physical threshold $u_{p}$ is a practical way to perform this identification. The independence of the identified events is checked using criteria such as minimal duration between events. An event has a certain duration that is typically longer than the time step of the series: this is why threshold exceedances in a time series occur in clusters. The events are then characterized by an event-describing random variable, most frequently their peak value. This step results in the extraction of an i.i.d. sample from the auto-correlated time series. Note that the event-describing variable and the sequential observations are two different random variables (e.g. peak Hs vs. hourly Hs). In a second step, the Extreme Value Theory (EVT) is called upon for determining the statistical threshold $u_{s}$ above which the exceedances of the event-describing variable may be modelled by a proper statistical distribution such as the GPD.

In the multivariate case, setting up an i.i.d. multivariate sample from the time series may be the first source for headaches to the analyst. Generally speaking, three types of multivariate cases may be distinguished and we propose the following classification:

- Type A: a metocean process described by several parameters (e.g.: a sea state described by its significant wave height $\mathrm{Hs}$, its peak period $\mathrm{Tp}$, its peak direction $\theta \mathrm{p}$, its directional spreading...);

- Type B: a metocean process that can be broken down in several elementary processes (e.g. a sea state made of a swell system and a wind sea system; or the sea level made of a mean sea level, the astronomical tide, the meteorological surge, the wave set-up...);

- Type C: the joint occurrence of several distinct metocean processes (e.g. waves, sea level, wind, current).

In this paper we focus on Type $\mathrm{C}$ analyses. Though an analysis of the physics of the phenomena and a Peaks-Over-Threshold approach provides a very practical framework in the univariate case, it is much more difficult in the multivariate case of Type $\mathrm{C}$. A combination of high level and moderate waves may cause overtopping, for instance: it is thus necessary to select data for both variables representative of both usual and rare conditions, while granting the independence of data values. Hence the conceptual approach of threshold exceedances must be adapted.

A first approach in the literature is specific to the joint occurrence of waves and sea levels, especially in tide-dominant areas. It is based on the postulate that for coastal structures, flooding or overtopping will only occur at high water. Consequently, the high-tide sampling is quite straightforward: the sea level at high tide (or closest to) is selected along with the associated Hs. Thus only one record per tidal cycle is extracted (i.e. nearly 2 per day). It is thus assumed that the lag between two successive high waters is long enough to consider that two successive records are independent. It seems acceptable for areas where the tide is diurnal (24h50mn between two high waters); it is probably a rather strong assumption for semi-diurnal areas $(12 \mathrm{~h} 25 \mathrm{mn})$. Nonetheless, this method is used in JOIN-SEA along with a recommendation to separate the data in two or three separate populations if needed for dealing with different sectors, seasons, wave systems....

Another approach, more directly linked to the POT declustering framework, consists of using a multivariate threshold. Li et al. (2014) applied a bivariate threshold for selecting 4-uplets of Hs, Tp, sea level $\mathrm{Z}$ and storm duration. An event (i.e. a storm) is defined when both Hs and the tidal anomaly TA (the meteorological surge) exceed a threshold value. To guarantee the independency of selected storms, a minimum time interval between two events is set. The following parameters are selected for setting up the i.i.d. sample: the maximum $\mathrm{Hs}$ value over the storm, the associated $\mathrm{Tp}$ value, the maximum sea level over the storm and the event duration (including possible fluctuations). This sampling has the 
advantage of being event-based, a framework that allows for instance to work on storm duration and to set the parameters granting independence the most adapted to the site and to the physical phenomenon; it is illustrated in Figure 1 that comes from Li et al. (2014).

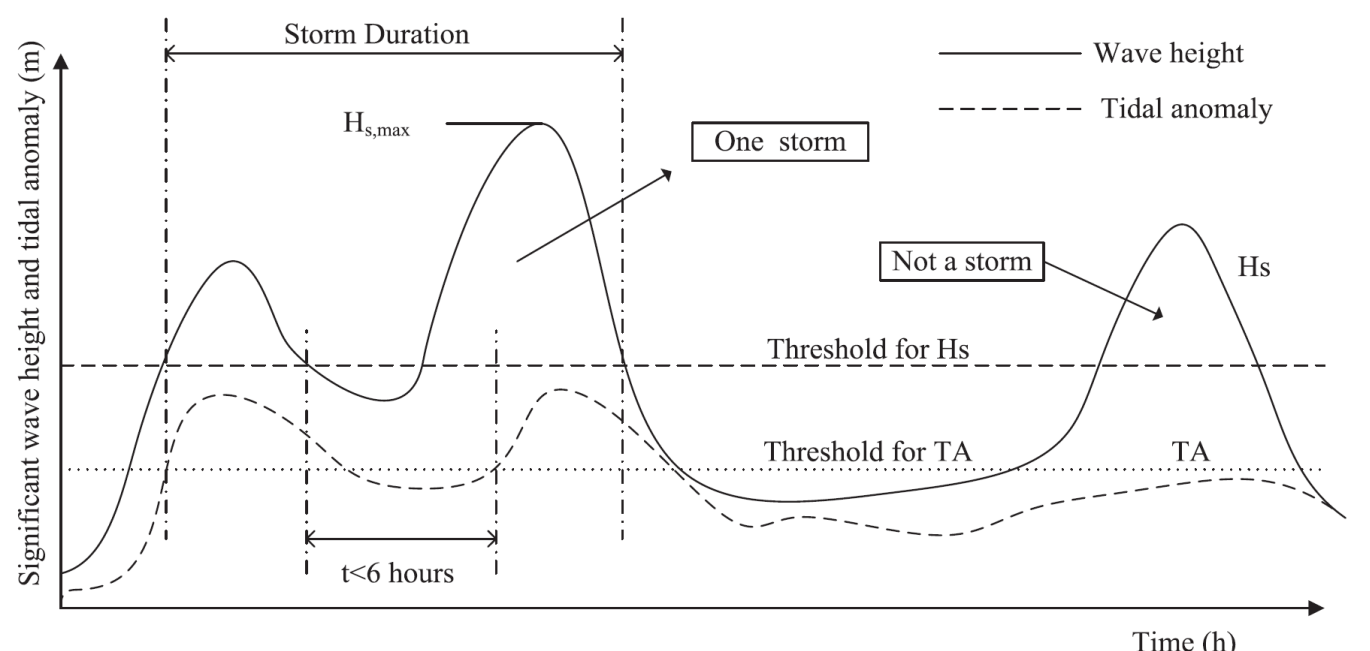

Figure 1: Definition of independent storm events by a bivariate threshold, after Li et al. (2014)

We propose here to test a new sampling method. In contrast to the bivariate threshold method presented above that focuses on the events of the input variables, we consider the effect of the combination of variables while reducing them into a univariate response variable. The notion of event is shifted from the input to the output. This function can be overtopping over a breakwater, efforts on a seawall, beach erosion, etc.... A classical POT declustering can then be applied and the peak of the univariate response function gives the date and time of the event: the i.i.d. sample is made of the n-uplets of the variables corresponding to these timestamps. The main advantage of this sampling is that covariates can be accounted for. Some phenomena are very sensitive to these covariates, or to characteristics of the events such as storm duration or inter-storm interval (e.g. for beach erosion, see Callaghan et al. 2008). Figure 2 below illustrates the very simple case of "total water level": the sum of sea level and wave height. The "total water level events" are identified by the exceedance of a threshold set at $12 \mathrm{~m}$ and characterized by their peak. The bivariate sample is made of the pairs (Hs, Z) corresponding to these peaks.

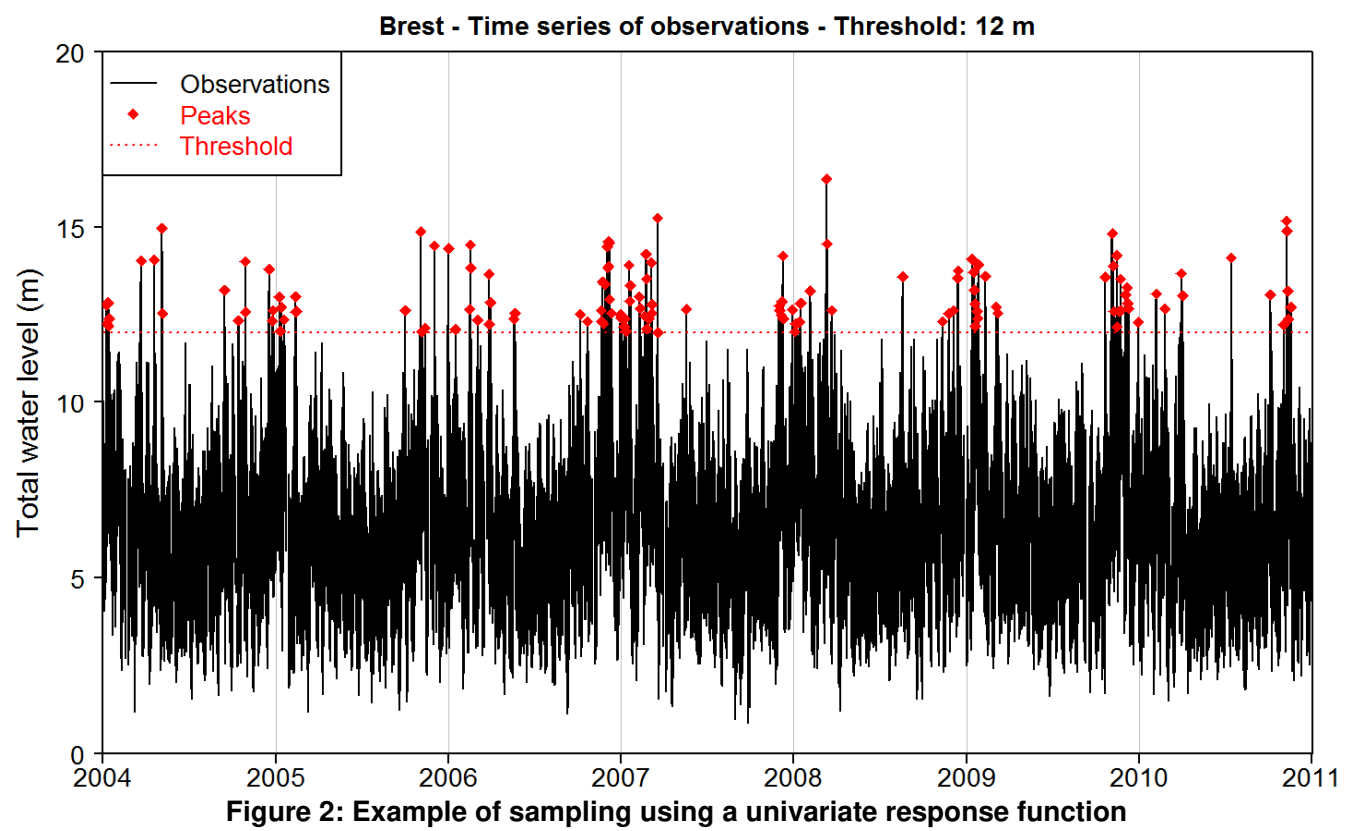

In the univariate case, Mazas and Hamm (2011) recommended setting the threshold so as to select 5 to 10 storms per year in average (in mid-latitude areas such as Western Europe). In a bivariate 
analysis, analysing and modelling the dependence requires more information and it is suggested to increase this number around 15 .

\section{Marginal distributions}

The combination of a large sea level with a moderate wave height may yield an extreme overtopping; a very large wave load may be generated by the association of a very high wave height with a frequent sea level on a seawall. So the marginal distributions should be able to represent both the extreme (tail) and frequent (bulk) values. Mixture models are convenient for this: the bulk is modelled by an empirical distribution while a parametric law (e.g. GPD) extrapolates the tail. Such a mixture model is used for surge distribution by Mazas et al. (2014).

\section{Dependence structure}

Several tools exist for analysing the dependence between two variables. The simplest is the scatterplot for visual analysis. Classical correlation coefficients such as Pearson's r, Spearman's $\rho$ or Kendall's $\tau$ coefficients, but they do not focus on tail dependence for extreme values.

The Chi-Plot $(\Lambda \mathrm{i}, \mathrm{Xi})$ introduced by Fisher and Switzer (1985) is a graphical tool for detecting dependence. $\mathrm{X}$ and $\Lambda$ are data transforms defined as a combination of the sample bivariate and marginal distribution functions. $\Lambda \mathrm{i}$ measures the distance of the pair $(\mathrm{Xi}, \mathrm{Yi})$ from the pair of the medians $(\bar{X}, \bar{Y})$ : a positive (resp. negative) value on the $\mathrm{X}$-axis of the chi-plot means that both $\mathrm{Xi}$ and $\mathrm{Yi}$ are on the same (resp. opposite) side of their respective medians, and a value close to 1 (resp. 0) means that are large or small relative to (resp. close to) their respective medians. Xi measures the dependence: a positive (resp. negative) value on the y-axis describes a positive (resp. negative) dependence, while a value close to zero suggests independence. Thus, it can be seen whether the dependence varies from the mean values to the tail regions.

The upper tail dependence coefficient (UTDC) $\lambda_{U}$ introduced by Sibuya (1960) describes the limit probability that a variable exceeds a threshold when the other does the same. $\lambda_{U}$ approaches 0 for independence and 1 for complete dependence. It provides the analyst with a coefficient dedicated to the area of interest, which will be linked to the copulas used for modelling the dependence structure. The CFG (Capéraà-Fougères-Genest) estimator (Capéraà et al. 1997) can be used for estimating $\lambda_{U}$ from the sample.

The dependence structure is modelled using parametric extreme value copulas. A copula is a multivariate probability distribution used to describe the dependence between random variables. According to Sklar's theorem, the joint cumulative distribution function $H_{X, Y}(x, y)=P[X \leq x ; Y \leq y]$ can be linked to the marginal distributions of $\mathrm{X}$ and $\mathrm{Y}, \mathrm{F}_{\mathrm{X}}$ and $\mathrm{F}_{\mathrm{Y}}$, via a copula $C$ :

$$
H_{X, Y}(x, y)=C\left(F_{X}(x), F_{Y}(y)\right)(1)
$$

Gudendorf and Segers (2010) provide a sound theoretical justification for using extreme-value copulas in the domain of extreme value multivariate analysis. In particular, a key advantage of the extreme value copulas with respect to the popular class of the Archimedean copulas is that they are not symmetric. Parametric extreme-value copulas include in particular the Gumbel-Hougaard (or logistic), Galambos (or negative logistic) and Hüsler-Reiss copulas. These three copulas are parameterized by a single dependence parameter $\theta$, whose value set the cursor between independence and complete dependence.

This parameter may be inferred from the sample using different estimators among which is the Canonical Maximum Likelihood (CML) estimator (Genest et al. 1995). The analytical relationship that exists between the UTDC and the dependence parameter may also be used for estimating $\theta$.

Last, a statistic of Cramér-von Mises may be used for testing the goodness of fit and choosing the best copula fit.

\section{Return periods associated to joint exceedance probabilities}

The joint exceedance probabilities can be computed from the copula function and the marginal cumulative distribution functions:

$$
P[X>x ; Y>y]=1+C_{\bar{\theta}}\left(F_{X}(x), F_{Y}(y)\right)-F_{X}(x)-F_{Y}(y)(2)
$$

If $\lambda_{\mathrm{p}}$ is the mean number of events per year (i.i.d. sample size over time series duration), then the return period associated to this probability can be expressed as follows: 


$$
T(x, y)=1 /\left(\lambda_{p}\left[1+C_{\bar{\theta}}\left(F_{X}(x), F_{Y}(y)\right)-F_{X}(x)-F_{Y}(y)\right]\right)
$$

Contours of equal return periods can then be plotted in the $(X, Y)$ plane.

The outline of the bivariate methodology can thus be sketched as illustrated in Figure 3.
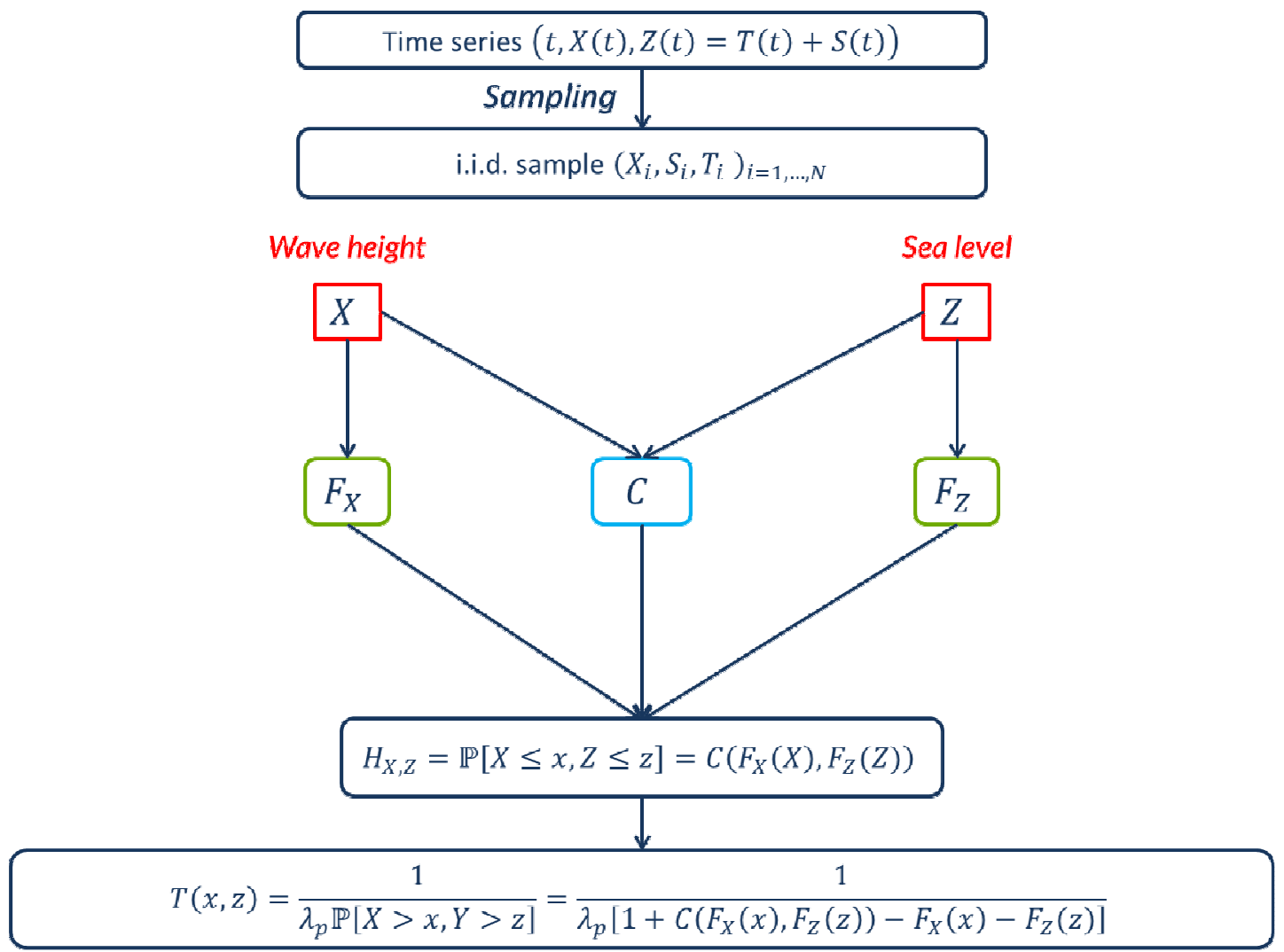

Figure 3: Outline of the bivariate methodology

\section{CASE STUDY}

\section{Presentation of the dataset}

The methodology is applied to a dataset of hourly sea levels and sea states off Brest, France (Figure 4) from 1953/01/01/ to 2010/12/31 (58 years, 501,363 sequential values for each variable). Sea levels Z come from the tide gauge of Brest operated by the French oceanographic service SHOM, whose software SHOMAR was used to compute the retropredictions of astronomical tide, which in turn allowed the extraction of the meteorological component (surge) S. Sea states came from a WaveWatchIII wave model set up by the University of La Rochelle, France. 


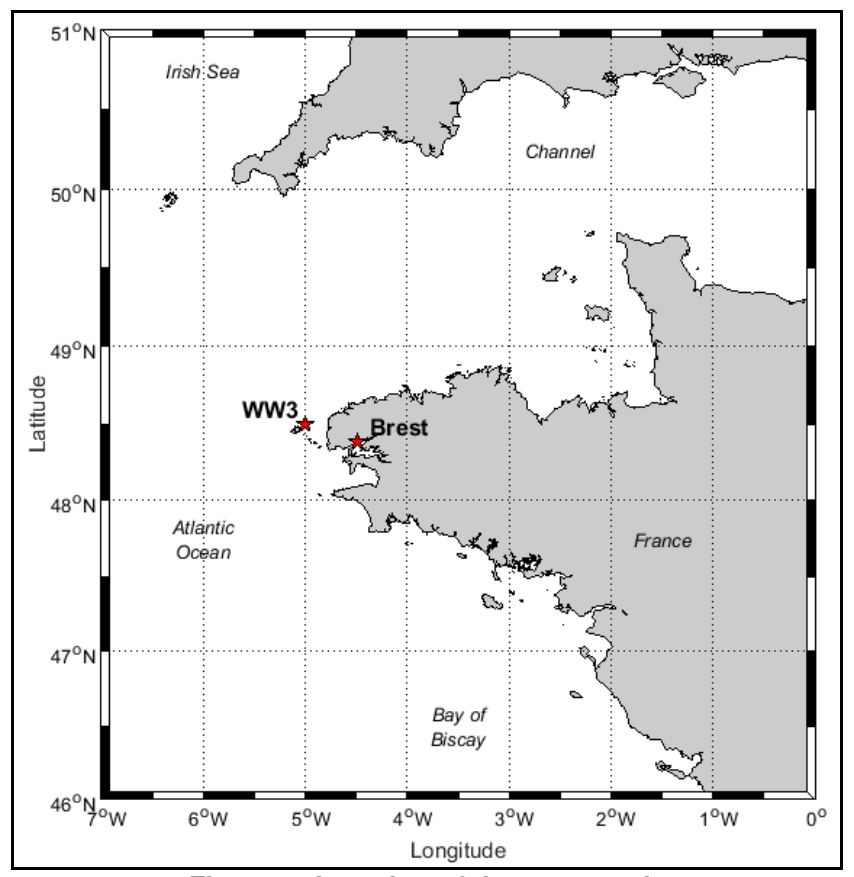

Figure 4: Location of the case study

\section{Application of the bivariate methodology}

A high tide sampling is performed. In order to reduce the sample (Hs, Z) so that JOIN-SEA can process it, a sub-sample with $\mathrm{Hs}>4.12 \mathrm{~m}$ is extracted, resulting in 5,753 values (Figure 5).

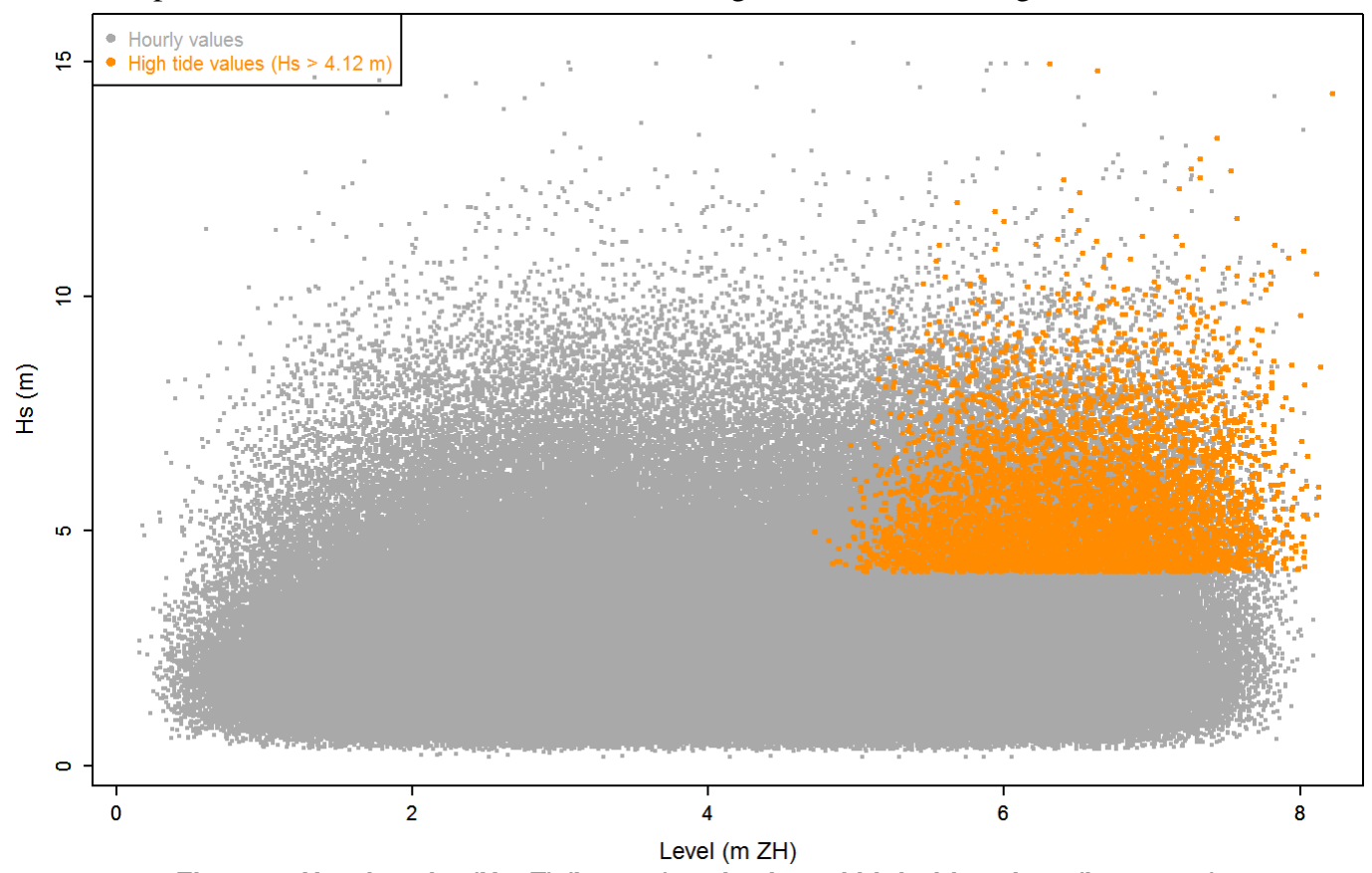

Figure 5: Hourly pairs (Hs, Z) (in grey) and selected high-tide values (in orange)

The marginal distributions of $\mathrm{Hs}$ and $\mathrm{Z}$ are modelled respectively by a GPD and an exponential distribution. As regards the dependence structure, the upper tail dependence coefficient of the sample is weak: 0.069. The Chi-plot confirms a weak positive dependence (Figure 6). 


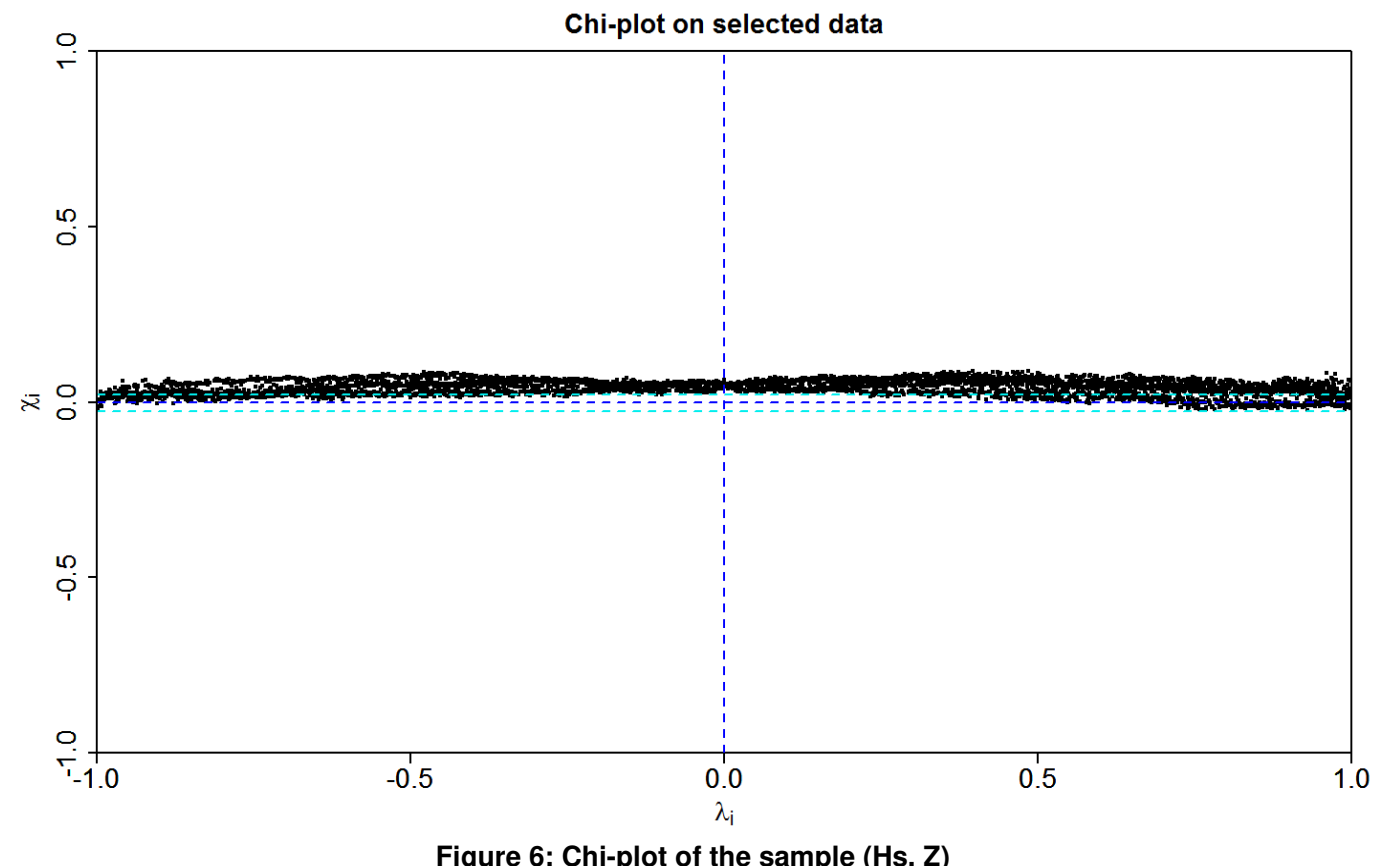

Figure 7 illustrates the fit of the three extreme value copulas estimated by both CML and UTDC methods. The Gauss copula is shown for illustrative purposes. The statistic of Cramér-von Mises show that the three extreme value copulas (Gumbel, Galambos, Husler-Reiss) are very close in terms of goodness-of-fit. The Galambos copula fitted by UTDC is chosen.

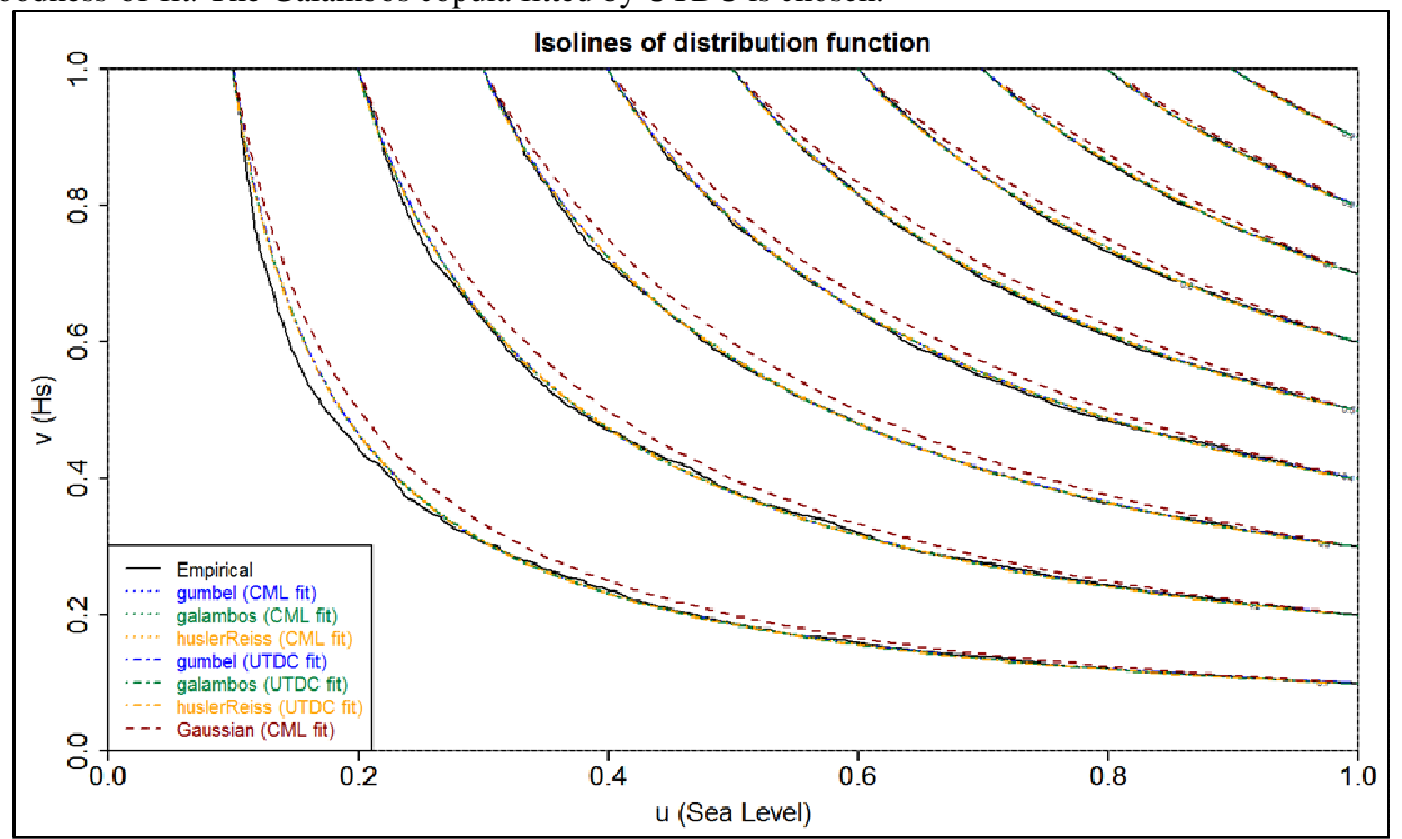

Figure 7: Comparison of empirical copula with extreme value copulas estimated by CML and UTDC and Gaussian copula fitted by CML (bivariate methodology)

Iso-contours of joint return periods of (Hs, Z) can then be drawn for a set of return periods (Figure 8 , blue lines). 


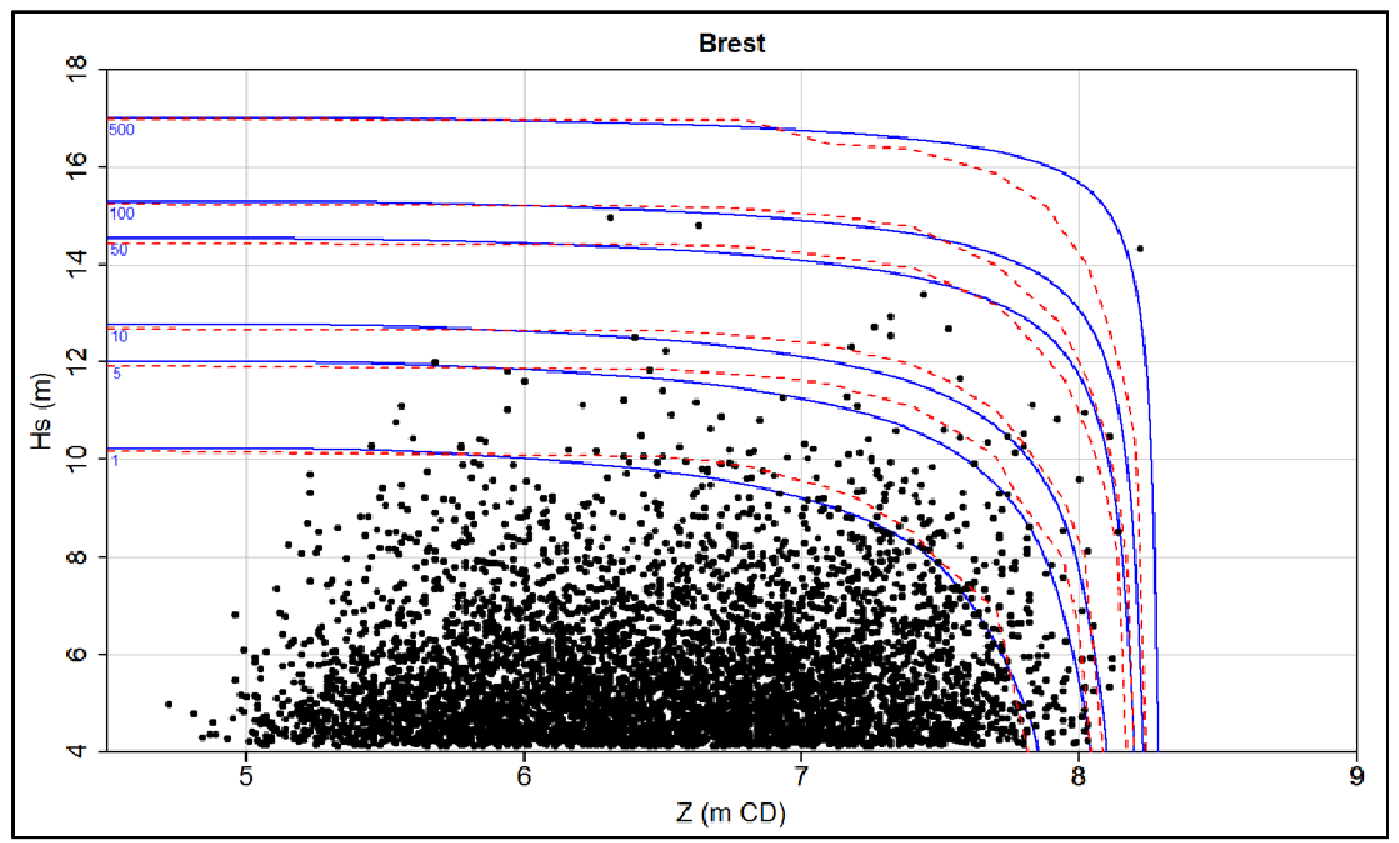

Figure 8: Comparison of joint return periods between the join-sea simulations (red dashed lines) and the bivariate methodology (blue plain lines)

\section{Comparison with results from JOIN-SEA}

The results from JOIN-SEA, kindly provided by Dr Hawkes, are shown in Figure 7 along with the results of the bivariate methodology. There is fair agreement for the marginal distributions (especially for Hs), but less good agreement for the dependence in the upper tail of the distribution. While JOINSEA is constrained to assume a constant level of dependence above a chosen threshold (with models such as the Bivariate Normal Distribution or Gaussian copula), the use of an extreme-value copula such as Gumbel-Hougaard copula makes the dependence between large wave heights and high sea levels continue to increase in the upper tail. It is to be noted that the correlation coefficient calculated by JOIN-SEA for the source data shows the coefficient to be still increasing into the upper tail: the use of extreme value copulas thus seems to be an appropriate approach for this dataset.

\section{EXTENSION TO A MULTIVARIATE METHODOLOGY}

\section{Outline of the methodology}

The case study above shows the weakness of the dependency between wave height and sea level in a strongly macrotidal environment. Thus the idea of considering separately astronomical tide and meteorological surge arises naturally: since the surge and the wave heights are often generated by the same weather system, the dependence between these two parameters should be stronger.

In addition, separating tide and surge also allows for a better estimation of extreme sea levels (Haigh et al 2010). The authors have proposed in Mazas et al. (2014) a POT-JPM approach for applying the event-based POT-declustering to the Joint Probability Method for extreme sea levels.

The sketch of the multivariate methodology is presented in Figure 9. A classical bivariate analysis between wave height $\mathrm{X}$ and surge $\mathrm{S}$ is performed in order to get their joint distribution $H_{X, S}$. Besides, a convolution between tide $\mathrm{T}$ and surge $\mathrm{S}$ yields the marginal distribution of sea level $\mathrm{Z}$. A "2D1D convolution" between the joint distribution of wave height and surge on the one hand and the distribution of tide on the other hand yields the joint distribution of wave height and sea level $H_{X, Z}$, whose combination with the marginal distributions $F_{X}$ and $F_{Z}$ gives the joint exceedance probabilities $P[X>x ; Z>z]$ that can be expressed in terms of joint return period $\mathrm{T}(\mathrm{x}, \mathrm{z})$. 


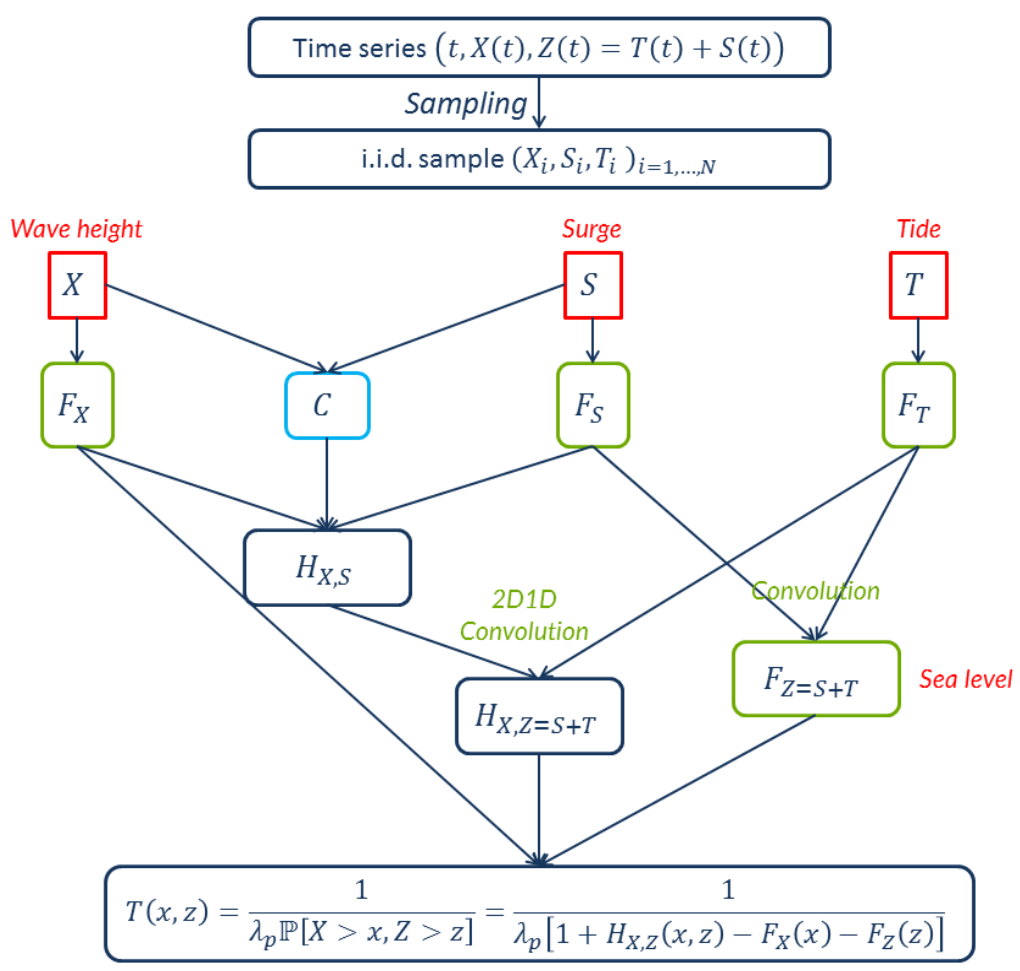

Figure 9: Outline of the multivariate methodology

\section{Application to the case study}

A sample based on the univariate response function "total water level" $(\mathrm{Z}+\mathrm{Hs})$ is used, as illustrated in Figure 2. A physical threshold $u_{p}=12 \mathrm{~m}$ is chosen, yielding an i.i.d. sample of 1082 tuples (Z, S, T, Hs), i.e. 18.7 events per year in average (Figure 10).

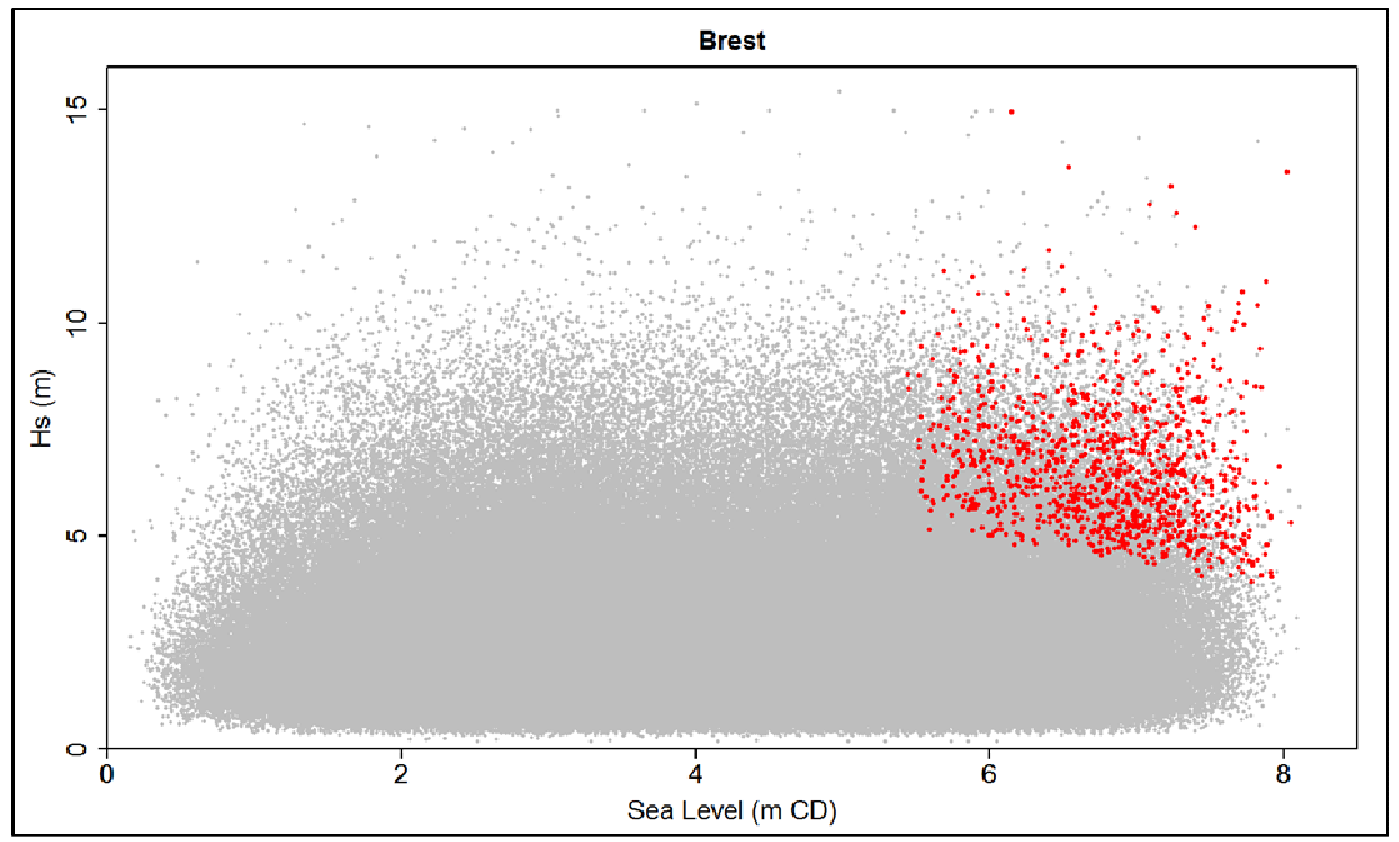

Figure 10: Hourly pairs (Hs, Z) (in grey) and i.i.d. sample of events (in red)

A GPD is chosen for modelling the upper tail of the marginal distributions of $\mathrm{S}$ and an exponential distribution for Hs. The tail distributions are connected to the bulk of the distributions modelled by the empirical distribution of the observations. The astronomical tide $\mathrm{T}$ is modelled by the empirical distribution of the 1,082 observations from the i.i.d. sample. A convolution then yields the distribution of the sea level. 
The dependence is now analyzed between Hs and the surge component S. It is illustrated by the chi-plot presented in Figure 11. The positive association is much more apparent than between Hs and $\mathrm{Z}$. The upper tail dependence coefficient $\lambda_{U}$ of the sample is logically much larger: 0.231 .

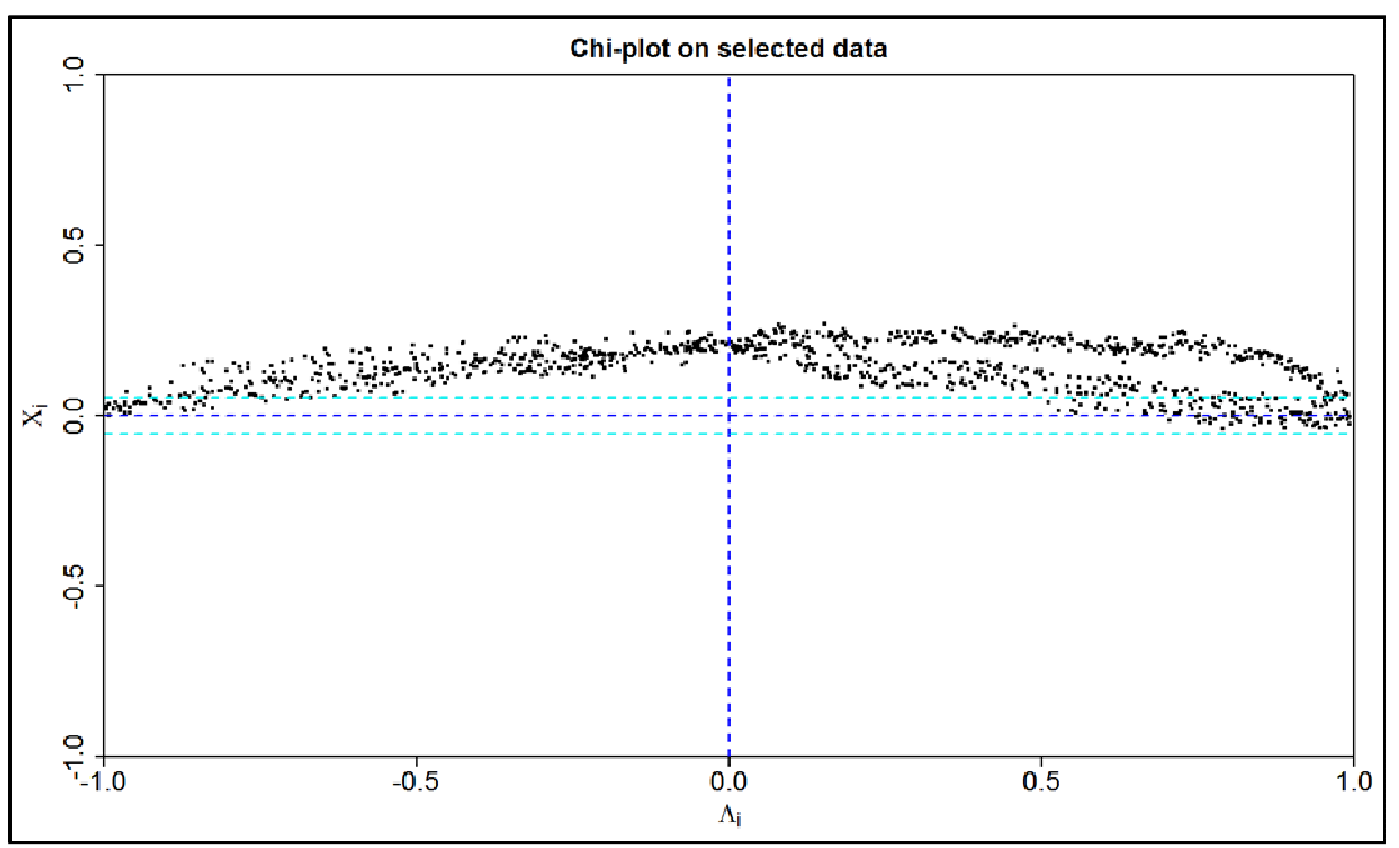

Figure 11: Chi-plot Hs/surge of the iid sample (multivariate methodology)

Once again, the three extreme value copulas, estimated by either the CML estimator or using the UTDC, are very close. Figure 12 shows that they all are much closer to the empirical copula than the classical Gauss copula. The CML-estimated Galambos copula is chosen as it minimizes the CVM statistic.

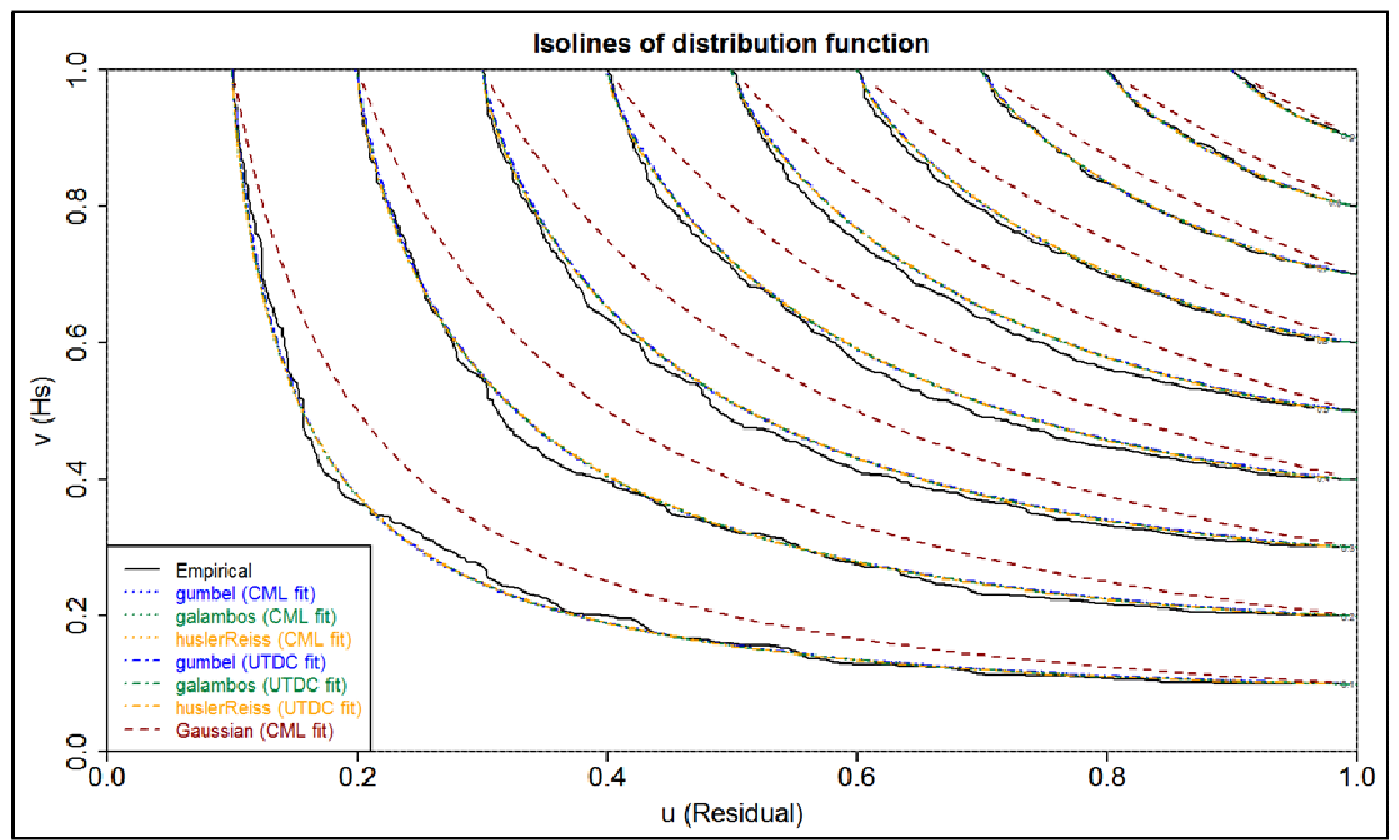

Figure 12: Comparison of empirical copula with extreme value copulas estimated by CML and UTDC and Gaussian copula fitted by CML (multivariate methodology)

The contours of equal return period associated to the joint exceedance probabilities of $\mathrm{Hs}$ and $\mathrm{Z}$ are provided in Figure 13. 


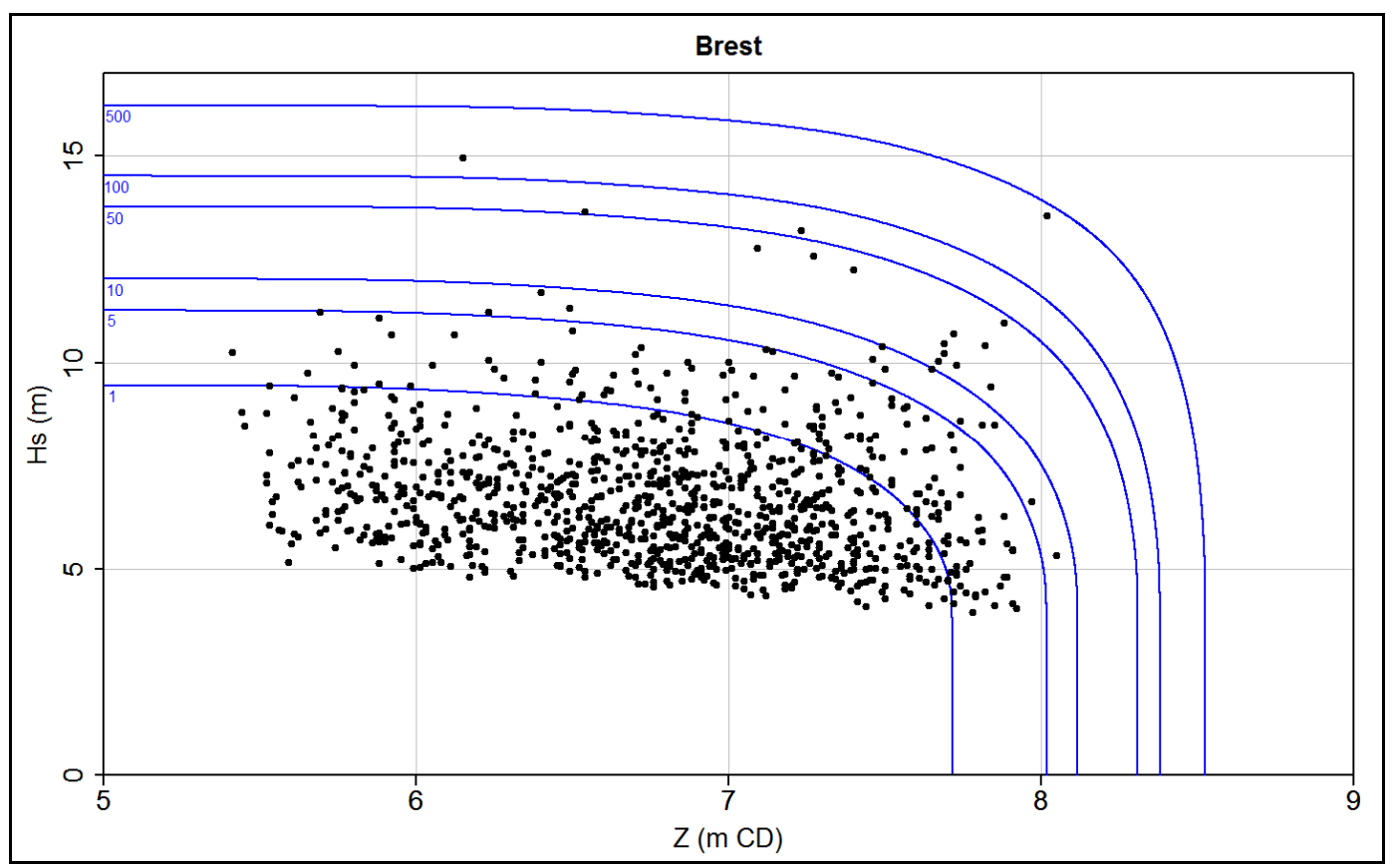

Figure 13: Joint return periods of wave height and sea level (multivariate methodology)

\section{DISCUSSIONS}

The use of extreme value copulas provide the analyst with mathematical functions that are adapted for modelling the structure of the dependence between two variables, particularly when the study aims at determining joint extremes. In particular, they allow for a varying level of dependence in the upper tail of the joint distribution.

The multivariate methodology presented in this paper allows for a proper estimation of extreme sea levels $\mathrm{Z}$ in tide-dominant areas. Indeed, the extreme sea levels resulting from this methodology (marginal distribution for Z) are much closer to the results of Mazas et al. (2014), who illustrated the POT-JPM methodology with the same case study: the difference is just $0.03 \mathrm{~m}$ for the 1-in-100-year sea level. In contrast, the bivariate methodology applies a simple direct extrapolation to the sea levels that yields an underestimation of extreme values (Haigh et al. 2010): the 1-in-100-year sea level is found to be $0.15 \mathrm{~m}$ lower than with the multivariate methodology.

A second advantage of this new multivariate methodology is that the dependence between surge and wave height is much stronger than between total sea level and wave height, at least for tidedominant areas such as Brest. This can be seen on the chi-plots, the sample upper tail dependence coefficients and the values of the copula parameter $\theta$ that takes values close to the independence case when considering $\mathrm{Hs}$ and $\mathrm{Z}$ directly, whereas $\theta$ notably increases in the multivariate approach. Modelling the dependence between surge and wave height has more sense and in more consistent with the physics.

Sampling is most probably the hardest issue when determining joint probabilities. Sampling must be linked to the concept of event, which needs to be defined in a multivariate case. It was highlighted in Bernardara et al. (2014) in the univariate case that the i.i.d. sample is made of a different random variable than the time series of sequential observations (e.g. Hs storm peak is a different random variable from hourly $\mathrm{Hs}$ ). This is particularly visible for multivariate analysis of Type $\mathrm{C}$ : the statistical model is applied to pairs (or tuples) describing the events identified by the sampling. In our case study, we have thus determined the joint probabilities of sea levels and offshore waves which result in a noticeable "total water level" nearshore, as defined by the univariate response function used for the sampling. It is obvious that other response functions, high tide sampling or the bivariate threshold sampling would give different results. First, it must be made clear that sampling by a univariate response function has both its pros (it accounts for covariates; it reduces to the univariate case which makes it easy to select independent tuples; different functions can be chosen or even combined) and cons (it is quite difficult to find a function that represents moderate and high values for both variables; results may change significantly from one function to the other). The aim of the present paper is to introduce it as an additional tool available for the analyst. Indeed, it is the responsibility of the analyst 
to choose the sampling method that is the most adapted to his analysis and there is no universal solution.

Last, the interest of a bivariate or multivariate methodology when the design focuses on a single univariate response function such as overtopping or beach overwash may be questioned. Wouldn't it be more efficient to compute directly the time series of overtopping from the original time series of sea level and wave height, period and direction, before extrapolating the result? Different answers can be made to this question. First, the analytical formulas used for computing such complex and non-linear phenomena may be unable to cover complicated situations caused by complex bathymetry or coastal structures. In that case, the formulas provide a rough estimate that is sufficient for the sampling stage, then the multivariate methodology provides the contours of equal joint exceedance probability that can be used for defining a small set of input conditions for physical modelling in a wave basin or detailed numerical modelling. In that regard, the multivariate methodology may be useful for overcoming practical difficulties.

Still the respective pros and cons of the univariate vs. multivariate approaches cannot be properly comprehended without a full understanding of the concepts of return period and event. It has already been shown by others (Hawkes et al. 2002) that the return period associated to the response function is lower than the joint return period associated to the source variables.

This means that when defining design criteria, it must be decided whether the return period (and hence the encounter probability, which is the truly important indicator) is applied to the source variables or to the response function(s). An example for the former case may be the estimation of meteo-oceanic conditions (wind, waves, levels, currents) in an offshore wind farm for which multivariate analyses are useful for many purposes from choice of location to structural design. This leads us back to our definition of events: are they related to environmental conditions or to structural response, to what comes or to what results?

Hence it should always be made clear in engineering whether the design criteria are to be based upon the source variables or the response function. This will drive the choices of the event, sampling and possibly methodology. This is the responsibility of the analyst to choose the sampling that is the most adapted to the meteo-oceanic environment and to the final aim of his study. In particular, a clear understanding of the concept of event and return period is key to a proper definition of design criteria for an engineering application.

\section{REFERENCES}

Bernardara P., F. Mazas, X. Kergadallan, and L. Hamm. 2014. A two-step framework for overthreshold modelling of environmental extremes, Nat Hazards Earth Syst Sci 14, 635-647.

Callaghan D.P., P. Nielsen, A. Short, and R. Ranasinghe. 2008. Statistical simulation of wave climate and extreme beach erosion, Coastal Engineering 55, 375-390.

Capéraà P., A.L. Fougères, and C. Genest. 1997. A nonparametric estimation procedure for bivariate extreme value copulas, Biometrika, 843, 567-577.

Fisher N. I. and Switzer P. 1985. Chi-plots for assessing dependence, Biometrika 72, 253-265.

Genest C., K. Ghoudi, L.P. Rivest (1995) A semiparametric estimation procedure of dependence parameters in multivariate families of distributions, Biometrika 82 (3), 543-552.

Gudendorf G. and J. Segers. 2010. Extreme-Value Copulas, in: Jaworski P., F. Durante, W.K. Härdle, and T. Rychlik (Eds.), Copula Theory and Its Applications, Lecture Notes in Statistics, Springer Berlin Heidelberg, pp. 127-145.

Haigh I.D., R. Nicholls and N. Wells. 2010. A comparison of the main methods for estimating probabilities of extreme still water levels, Coastal Engineering 57, 838-849.

Hawkes P.J., B.P. Gouldby, J.A. Tawn, and M.W. Owen. 2002. The joint probability of waves and water levels in coastal engineering design, Journal of Hydraulic Research 40, 241-251.

Li F., P.H.A.J.M. van Gelder, R. Ranasinghe, D.P. Callaghan, and R.B. Jongejan. 2014. Probabilistic modelling of extreme storms along the Dutch coast, Coastal Engineering 86, 1-13.

Mazas F. and L. Hamm. 2011. A multi-distribution approach to POT methods for determining extreme wave heights, Coastal Engineering 58, 385-394.

Mazas F., X. Kergadallan, P. Garat, and L. Hamm. 2014. Applying POT methods to the Revised Joint Probability Method for determining extreme sea levels, Coastal Engineering 91, 140-150.

Sibuya M. 1960. Bivariate extreme statistics, Ann. Inst. Statist. Math., 11:195-210.

Walton, T.L. 2000. Distribution for storm surge extremes, Ocean Engineering 27, 1279-1293. 